\title{
Machine Learning Techniques for Sequence-based Prediction of Viral-Host Interactions between SARS-CoV-2 and Human Proteins
}

\author{
Lopamudra Dey, Sanjay Chakraborty, Anirban Mukhopadhyay \\ Dept. of Computer Science $\mathcal{E}$ Engineering, Heritage Institute of Technology, Kolkata, India \\ Dept. of Information Technology, Techno India, Saltlake, Kolkata, India \\ Dept. of. Computer Science $\&$ Engineering, University of Kalyani, Kayani, India \\ Email: anirban@klyuniv.ac.in
}

\begin{abstract}
COVID-19 (Coronavirus Disease-19), a disease caused by the SARS-CoV-2 virus, has been declared as a pandemic by the World Health Organization on March 11, 2020. Over 4.3 million people from more than 200 countries have already been affected throughout the world by this deadly virus, resulting in almost 0.3 millions deaths. Protein-protein interactions (PPIs) play a key role in the cellular process of SARS-CoV-2 virus infection in the human body. Recently a study has reported some SARS-CoV-2 proteins that interact with a number of human proteins while many potential interactions still remain to be identified. However, human cells are composed of a large number of proteins. Therefore, it is not possible to experimentally check all possible combinations of interactions. This leads to development of various computational methods to predict the PPIs between the virus and human proteins and further validation of them using biological experiments. This paper presents a prediction model by combining the different sequence-based features of human proteins like the amino acid composition, pseudo amino acid composition, and the conjoint triad. We have built an ensemble voting classifier using $S V M^{\text {Radial }}, S V M^{\text {Polynomial, }}$, and Random Forest technique which gives greater accuracy, precision, specificity, recall, and F1 score over all other models used in the work. We have predicted 1326 potential human target proteins using this weighted ensemble classifier. Furthermore, the Gene Ontology (GO) and KEGG pathway enrichments of these predicted human proteins are investigated. This study may encourage the identification of potential targets for more effective anti-COVID drug discovery.
\end{abstract}

Keywords: COVID-19, SARS-CoV-2, Protein-Protein Interaction, Supervised Classification, Machine Learning, Classifier Ensemble. 